\title{
Finite Element Analysis with Iterated Multiscale Analysis for Mechanical Parameters of Composite Materials with Multiscale Random Grains
}

\author{
Youyun Li, ${ }^{1,2}$ Yuqing Pan, ${ }^{1}$ Jianlong Zheng, ${ }^{1}$ \\ Chiqing Zhou, ${ }^{1}$ and Desheng Wang ${ }^{3}$ \\ ${ }^{1}$ School of Traffic and Transportation Engineering, Changsha University of Science and Technology, Hunan \\ 410004, China \\ ${ }^{2}$ State Key Laboratory of Structural Analysis for Industrial Equipment, Dalian University of Technology, \\ Dalian 116024, China \\ ${ }^{3}$ Division of Mathematical Sciences, School of Physical and Mathematical Science (SPMS), Nanyang \\ Technological University, 21 Nanyang Link, Singapore 637371
}

Correspondence should be addressed to Youyun Li, liyouyun8@hotmail.com

Received 11 August 2010; Revised 22 December 2010; Accepted 17 March 2011

Academic Editor: Moran Wang

Copyright (C) 2011 Youyun Li et al. This is an open access article distributed under the Creative Commons Attribution License, which permits unrestricted use, distribution, and reproduction in any medium, provided the original work is properly cited.

\begin{abstract}
Based on the iterated statistically multiscale analysis (SMSA), we present the convergence of the equivalent mechanical parameters (effective moduli), obtain the error result, and prove the symmetric, positive and definite property of the equivalent mechanical parameters tensor computed by the finite element method. The numerical results show the proved results and illustrate that the SMSA-FE algorithm is a rational method for predicting the equivalent mechanical parameters of the composite material with multiscale random grains. In conclusion, we discuss the future work for the inhomogeneous composite material with multiscale random grains.
\end{abstract}

\section{Introduction}

Predicting the mechanical parameters of a composite material with the multiscale random grains is a very difficult problem because there are too many random grains in the composite material and the range of the scale of the grains is very large in the material field shown in Figure 1. Many studies on predicting physical and mechanical properties of composite materials with random grains have been done: the law of mixtures [1], the HashinShtrikman bounds [2], the self-consistent method [3], the Eshelbys equivalent inclusion method [4] and the Mori-Tanaka method [5], microanalysis method [6], and so forth. These methods effectively promoted the development of composite materials, but they simplified 
the microstructure of real materials in order to reduce the computational complexity. The composite materials with large numbers of grains can be divided into two classes according to the basic configuration: the composite materials with periodic configurations, such as braided composites, and the composite materials with random distribution, such as concrete, foamed plastics. Some physical methods and mathematical methods [7-13] have been proposed to solve these problems. However, most of these techniques and methods are based on empirical, semiempirical models or based on the homogenization methods in the periodical structure. Due to the difference of basic configuration, it is difficult for them to handle the composite material with large numbers of multiscale random grains. Hence, in order to evaluate the physical and mechanical performance of the composite material with random grains, it is necessary to make use of the different advanced numerical methods.

In the recent decades, for the problems with the stationary random distribution, Jikov et al. [14] developed the homogenization method and proved the existence of the homogenization coefficients and the homogenization solution, however, not provided with the numerical techniques to carry out the methods for the stationary random distribution. In addition, their method only deals with the point randomly distributed, not with random grains.

For the porous medias with the random distribution, Hou and $\mathrm{Wu}$ [15] developed the the multiscale finite element base function method to compute these problems of the porous medias; this method is much valid to the problems with random coefficients and problems with continuous scale. As for the multiscale systems with stochastic effective, Vanden-Eijnden gave the specific step to carry the multiscale method out [16]; their methods are very effective to mainly solve the problem with time process. For the perforated domain with small holes, Wang et al. gave an effective macroscopic model for a stochastic microscopic system, and in theory, mainly proved that the solutions of the microscopic model converge to those of the effective macroscopic model in probability distribution as the size of holes diminishes to zero [17]. However, the above methods are not for the equivalent performance of the composite material with large numbers of the multiscale random grains.

Duschlbauer et al. developed the homogenization method with the Mori-Tanaka scheme averaging microfields extracted for individual fibers and the finite element analysis to estimate the linear thermoelastic and thermophysical behavior of a short fiber reinforced composite material with planar random fiber arrangement [18]. Kari et al. developed a representative volume element (RVE) approach that was used to calculate effective material properties of randomly distributed short fibre composites and analyzed the properties for the volume of random short fibres [19]. Recently, Kalamkarov et al. gave an asymptotic homogenization model for the 3D grid-reinforced composite structures with the orthotropic reinforcements [20,21], and Wang and Pan obtained the elastic property for the multiphase composites with the random microstructures [22, 23]. Their methods are the effective homogenous methods for the equivalent performance of the composites with the random grains. However, in fact, in the engineering fields, for the composite materials with a large number of multiscale random grains, such as concrete, asphalt mixture, rock mass, and foam plastics, owing to the random complexity in configuration and for that the grain scale range is very large from $10^{-1} \mathrm{~m}$ to $10^{-6} \mathrm{~m}$ [24], shown in Figure 1, the above methods find it difficult to analyze the mechanical and physical performance. Hence, in order to deal with the composite materials with multiscale random grains, authors proposed a kind of statistically multiscale analysis (SMSA) method to predict the effective mechanical parameters of the composite materials with a large number of random multiscale grains [2527]. In previous papers [25-27], we proposed an expression for predicting the equivalent 
mechanical parameters of a composite material with multiscale grains. This method cannot only show the macrocharacteristics and random configurations of a composite material, but also show the contribution of the small-scale grains. In addition, this method can greatly decrease the computing time for the required numerical result.

In these previous papers [25-27], we gave the multiscale formula to compute the composite material and proved that the expected displacement field is convergent to the equivalent displacement field based on the multiscale methods in the mathematical theory. However, we did not discuss the convergence, the error result, and the symmetric, positive definite property of the equivalent mechanical parameter tensor of the composite material with random grains by the SMSA-FE method. Therefore, in this paper, the convergence and the error result based on statistical multiscale analysis (SMSA) shall be presented, and the symmetric, positive definite property of the equivalent parameters tensor (the random parameters subjected to the uniform distribution) shall be proved.

The next section reviews a representation of a composite material with multiscale random grains, some results, and the SMSA-FE procedure [27]. Section 3 is devoted to proving the convergence of the equivalent mechanical parameters computed by the SMSA-FE algorithm. Section 4 obtains the error results of the SMSA-FE algorithm on iterated multiscale analysis. In Section 5, the symmetric, positive definite property of the equivalent mechanics parameters tensor computed by the SMSA-FE algorithm is proved. In Section 6, the numerical results are presented to demonstrate the validity, the convergence, and the proved results of the SMSA-FE algorithm. Finally, we discuss the future work for the inhomogeneous composites with multiscale random grains.

\section{Iterated Multiscale Analysis Model and Algorithm}

In the previous papers [27], the author had given the algorithm to compute the equivalent mechanical parameters in detail. In order to prove the finite element error and the convergence of the iterated multiscale computed model, we shall review the model and the algorithm in the brief.

\subsection{Iterated Multiscale Analysis Model}

For the brief, all of the grains are assumed as the ellipsoids. Set a domain $\Omega$ to represent a composite with multiscale random grains shown in Figure 1(a). Set $\Omega_{l}$ to be a set of cube cells of the size $\varepsilon^{l}$ shown in Figure 1(b). Based on [27], the iterated multiscale analysis model can be represented as follows.

(1) Obtain the statistical data of the composites and specify the distributions $P$ of the ellipsoid's parameters.

(2) Set $N$ to denote the number of the $l$ th scale ellipsoids in the cell $\varepsilon^{l} Q^{s}$; we can describe the $l$ th scale cube cell $\varepsilon^{l} Q^{s}$ as follows:

$$
\omega_{l}^{s}=\left(a_{1}^{s}, b_{1}^{s}, c_{1}^{s}, \theta_{a_{x_{1} x_{2}}}^{s}, \theta_{a_{x_{1}}}^{s}, \theta_{b_{x_{1} x_{2}}}^{s}, \theta_{b_{x_{1}}}^{s}, x_{1^{0}} \stackrel{s}{1_{1}}, x_{2^{0}}{ }_{1}^{s}, x_{3^{0}}{ }_{1}^{s} \ldots, \theta_{b_{x_{1}}}^{s}, x_{1^{0}}{ }_{N}, x_{2^{0}}{ }_{N}, x_{3^{0}}{ }_{N}\right),
$$

where $\left(x_{1^{0}}, x_{2^{0}}, x_{3^{0}}\right)$ is the center point, $a$ is the long axis, $b$ is the middle axis, $c$ is the short axis, and $\theta_{a_{x_{1} x_{2}}}, \theta_{a_{x_{1}}}, \theta_{b_{x_{1} x_{2}}}$ and $\theta_{b_{x_{1}}}$, are the directions for the axis $a$ and $b$ of the ellipsoids, respectively. One sample $\omega_{l}^{s}$ is shown in Figure 1(c). 


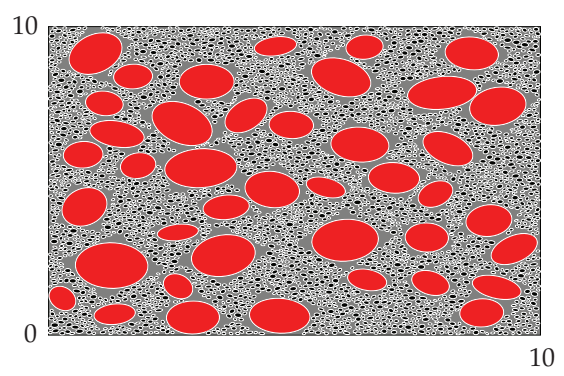

(a)

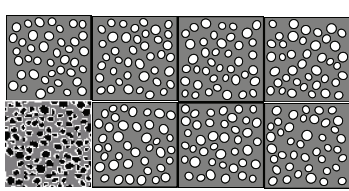

(b)

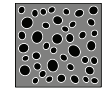

(c)

Figure 1: (a) $\Omega$ with multiscale grains, (b) equivalent matrix with random grains, (c) $\varepsilon^{2}$ cell.

(3) Set the domain $\Omega_{l}$ to be logically composed of $\varepsilon^{l}$-sized samples: $\Omega_{l}=$ $\bigcup_{\left(\omega^{s}, t \in Z\right)} \varepsilon^{l}\left(Q^{s}+t\right)$ shown in Figure $1(\mathrm{~b})$. It can be defined as $\omega_{l}=\left\{\omega_{l}^{s}, x \in \varepsilon Q_{l}^{s} \subset \Omega_{l}\right\}$. By the SMSA-FE algorithm [27], the equivalent mechanical parameters can be predicted. Thus, the equivalent material with $l$ th scale random grains in $\Omega_{l}$ can be formed.

(4) Set $m$ to be the scale number in the composites $\Omega$ with multiscale random grains. For $l=m-1, \ldots, 1$, using the above representation from step (2) to step (3), recursively and successively, the multiscale random model of $\Omega$ can be described.

For example, the asphalt concrete [24] can be considered as the composites with multiscale random grains, respectively. Set $\varepsilon^{2}=0.01 \mathrm{~m}$; and $\varepsilon^{1}=0.1 \mathrm{~m}$, their configuration can be shown in Figure 1.

\subsection{SMSA Algorithm Based on Finite Element Method}

In the previous section, we introduced the equivalent composites $\Omega_{l},(l=m, m-1, \ldots, 1)$ with the $l$ th scale grains. In this section, we shall review the mathematical theory that predicts the equivalent mechanical parameters of these composites with random grains by the statistical multiscale analysis (SMSA) [27].

For the domain $\Omega_{l},(l=m-1, m-2, \ldots, 0)$ shown in Figure $1(b)$, their corresponding elasticity equation system and the essential boundary condition can be shown as follows:

$$
\begin{aligned}
& \frac{\partial}{\partial x_{j}}\left[a_{i j h k}^{\varepsilon}\left(\frac{x}{\varepsilon}, \omega\right) \frac{1}{2}\left(\frac{\partial u_{h}^{\varepsilon}(x, \omega)}{\partial x_{k}}+\frac{\partial u_{k}^{\varepsilon}(x, \omega)}{\partial x_{h}}\right)\right]=f_{i}(x), \quad x \in \Omega_{l} \\
& u^{\varepsilon}(x, \omega)=\bar{u}(x), \quad x \in \partial \Omega
\end{aligned}
$$

where $i, j, h, k=1,2, \ldots, n, \omega=\omega^{s}$ for $x \in \Omega_{1}, \omega^{s} \in P, P$ is the probability space, $\Omega_{l}=$ $\bigcup_{s \in p, t \in Z^{n}} \varepsilon^{l}\left(Q^{s}+t\right)$ shown in Figure $1, u_{h}^{\varepsilon}(x, \omega)$ are the displacement field, $f_{i}(x)$ are the loads, and $\bar{u}(x)$ is the boundary displacement vector.

In the paper [25-27], we had given the SMSA method [25] and established the finite element method [27] to compute the equivalent mechanical parameters. If the FE space $\left(V^{h_{0}}\left(Q^{s}\right)\right)^{n}$ can be established and $\xi=x / \varepsilon^{l}$, the equivalent mechanical parameters can be computed. 
Theorem 2.1. If a composite material with random grains is subjected to the probability distribution $P$, the equivalent mechanical parameters of a composite material can be approximated as

$$
\widehat{a}_{i j h k}^{h_{0}}=\frac{\sum_{s=1}^{M} \widehat{a}_{i j h k}^{h_{0}}\left(\omega^{s}\right)}{M}
$$

where $\omega^{s} \in P(s=1,2, \ldots, M)$ and $\widehat{a}_{i j h k}^{h_{0}}\left(\omega^{s}\right)$ is computed as

$$
\begin{gathered}
\widehat{a}_{i j h k}^{h_{0}}\left(\omega^{s}\right)=\frac{1}{\left|Q^{s}\right|} \int_{Q^{s}}\left(a_{i j h k}\left(\xi, \omega^{s}\right)+a_{i p h q} \varepsilon_{p q}\left(N_{k j}^{h_{0}}\left(\xi, \omega^{s}\right)\right)\right) \mathbf{d} \xi, \\
\mathbf{N}_{\alpha}^{h_{0}}\left(\xi, \omega^{s}\right)=\left(\mathbf{N}_{\alpha 1}^{h_{0}}, \mathbf{N}_{\alpha 2}^{h_{0}}, \ldots, \mathbf{N}_{\alpha n}^{h_{0}}\right)=\left(\begin{array}{ccc}
N_{\alpha 11}^{h_{0}}\left(\xi, \omega^{s}\right) & \cdots & N_{\alpha 1 n}^{h_{0}}\left(\xi, \omega^{s}\right) \\
\vdots & & \vdots \\
N_{\alpha n 1}^{h_{0}}\left(\xi, \omega^{s}\right) & \cdots & N_{\alpha n n}^{h_{0}}\left(\xi, \omega^{s}\right) .
\end{array}\right) .
\end{gathered}
$$

$\mathbf{N}_{\alpha}^{h_{0}}\left(\xi, \omega^{s}\right)$ are the FE solutions of (2.6) on unit cell $Q^{s}$.

$$
\begin{gathered}
\frac{\partial}{\partial \xi_{j}}\left(a_{i j h k}\left(\xi, \omega^{s}\right) \varepsilon_{h k}\left(N_{\alpha m}\left(\xi, \omega^{s}\right)\right)\right)=-\frac{\partial a_{\alpha i l m}\left(\xi, \omega^{s}\right)}{\partial \xi_{l}}, \quad \xi \in Q^{s}, \\
\mathbf{N}_{\alpha}\left(\xi, \omega^{s}\right)=0, \quad \xi \in \partial Q^{s} .
\end{gathered}
$$

Therefore, the equivalent mechanical parameters can be determined by the following SMSA-FE algorithm.

SMSA-FE Algorithm

(1) Specify the scale number $m$ of random grains in the composites and set the iterative number $r=m$.

(2) Model $r$-scale random grains in $Q_{r}^{s}$ and generate meshes of the sample domain according to the algorithm in [27].

(3) If $r=m, a_{i j h k}\left(x / \varepsilon^{r}, \omega^{s}\right)$ in $Q_{r}^{s}$ can be indicated as follows:

$$
a_{i j h k}\left(\frac{x}{\varepsilon^{r}}, \omega^{s}\right)= \begin{cases}a_{i j h k^{\prime}}^{1} & x \in \varepsilon^{r} \widehat{Q}^{s}, \\ a_{i j h k^{\prime}}^{2} & x \in \varepsilon^{r} \widetilde{Q}^{s}, \\ \cdots & \\ a_{i j h k^{\prime}}^{l} & x \in \varepsilon^{r} \widehat{Q}^{s},\end{cases}
$$

where $\widehat{Q}^{s} \cup \widetilde{Q}^{s} \cup \cdots \cup \widehat{Q}^{s}=Q_{r}^{s}$ and $\widehat{Q}^{s} \cap \widetilde{Q}^{s} \cdots \cap \widehat{Q}^{s}=\phi, \varepsilon^{r} Q_{r}^{s} \subset \Omega$ denotes the domain of the basic configuration, $\varepsilon^{r} \widehat{Q}^{s}$ denotes the domain of the matrix, $\varepsilon^{r} \widetilde{Q}^{s}$ 
denotes the domain of the random grains in $\varepsilon^{r} Q_{r}^{s}$, and $\varepsilon^{\bar{a}} \widehat{Q}^{s}$ denotes the interface domain between the grains and the matrix. Go to step (5).

(4) If $r<m, a_{i j h k}\left(x / \varepsilon^{r}, \omega^{s}\right)$ in $Q_{r}^{s}$ can be indicated as follows:

$$
a_{i j h k}\left(\frac{x}{\varepsilon^{r}}, \omega^{s}\right)= \begin{cases}\widehat{a}_{i j h k}^{h_{0}}\left(\frac{x}{\varepsilon^{(r+1)}}, \omega^{s}\right), & x \in \varepsilon^{r} \widehat{Q}^{s}, \\ a_{i j h k^{\prime}}^{1} & x \in \varepsilon^{r} \widetilde{Q}^{s},\end{cases}
$$

where $\widehat{Q}^{s} \cup \widetilde{Q}^{s}=Q_{r}^{s}$ and $\widehat{Q}^{s} \cap \widetilde{Q}^{s}=\phi, \varepsilon^{r} Q_{r}^{s} \subset \Omega$ denotes the domain of the basic configuration, $\varepsilon^{r} \widehat{Q}^{s}$ denotes the domain of the equivalent matrix, and $\varepsilon^{r} \widetilde{Q}^{s}$ denotes the domain of random grains. Go to step (5).

(5) Compute the FE approximation $N_{\alpha m}^{h_{0}}\left(\xi^{r}, \omega^{s}\right)$ according to (2.6), obtain the FE approximation of sample $\hat{a}_{i j h k}^{h_{0}}\left(\varepsilon^{r}, \omega^{s}\right)$ on the $r$ th scale according to (2.4), and compute the FE approximation of the equivalent mechanical parameter tensor $\left\{\widehat{a}_{i j h k}^{h_{0}}\left(\varepsilon^{r}\right)\right\}$ on the $r$ th scale using (2.3).

(6) Set $\widehat{a}_{i j h k}\left(\varepsilon^{r}\right)$ equal to $\widehat{a}_{i j h k}^{h_{0}}\left(\varepsilon^{r}\right)$ on the $r$ th scale and $r=r-1$. If $r>1$, go to step (2). Otherwise, the equivalent mechanical parameter tensor $\widehat{a}_{i j h k}\left(\varepsilon^{r}\right)(\varepsilon)$ is the equivalent mechanical parameter tensor of the composite material with multiscale random grains in $\Omega$.

\section{Convergence of SMSA-FE Algorithm}

Lemma 3.1. If $N_{\alpha m}^{h_{0}}(\xi, \omega)$ and $\hat{a}_{i j h k}^{h_{0}}(\omega)$ are the finite element approximations of the random variables $N_{\alpha m}(\xi, \omega)$ and $\widehat{a}_{i j h k}(\omega)$, respectively, then there exists a constant $M_{2}>0$ such that $\left|\hat{a}_{i j h k}^{h_{0}}(\omega)\right|<M_{2}$.

Proof. If $\left|a_{i j h k}\left(\xi, \omega^{s}\right)\right|<M$ for any sample $\omega^{s} \in P$, (2.6) has one unique finite element solution $N_{\alpha m}^{h_{0}}\left(\xi, \omega^{s}\right) \in\left(H^{1}\left(Q^{s}\right)\right)^{n}(n=2,3)$ such that

$$
\left\|N_{\alpha m}^{h_{0}}\left(\xi, \omega^{s}\right)\right\|_{\left(H^{1}\left(Q^{s}\right)\right)^{n}}<C\left|a_{i j h k}\left(\xi, \omega^{\mathrm{s}}\right)\right|_{L^{\infty}\left(Q^{s}\right)}<C M_{1}
$$

where $C$ and $M_{1}$ are constants and independent of $\xi$ and $\omega^{s}$. 
From (2.4) and (3.1), one can obtain

$$
\begin{aligned}
& \left|\hat{a}_{i j h k}^{h_{0}}\left(\omega^{s}\right)\right| \\
& \quad=\left|\frac{1}{\left|\operatorname{mes}\left(Q^{s}\right)\right|} \int_{\left(Q^{s}\right)}\left(a_{i j h k}\left(\xi, \omega^{s}\right)+a_{i p h q}\left(\xi, \omega^{s}\right) \frac{1}{2}\left(\frac{\partial N_{k p j}^{h_{0}}\left(\xi, \omega^{s}\right)}{\partial \xi_{q}}+\frac{\partial N_{k q j}^{h_{0}}\left(\xi, \omega^{s}\right)}{\partial \xi}\right)\right) \mathrm{d} \xi\right| \\
& \quad<\frac{1}{\left|\operatorname{mes}\left(Q^{s}\right)\right|}\left(\left\|a_{i j h k}\left(\xi, \omega^{s}\right)\right\|_{L^{\infty}\left(Q^{s}\right)}+\left\|a_{i p h q}\left(\xi, \omega^{s}\right)\right\|_{L^{\infty}\left(Q^{s}\right)}\left\|N_{k j}\left(\xi, \omega^{s}\right)\right\|_{\left(H^{1}\left(Q^{s}\right)\right)^{n}}\right) \\
& \quad \leq \frac{1}{\left|\operatorname{mes}\left(Q^{s}\right)\right|}\left|M_{1}+M_{1} * C M_{1}\right|\left|\operatorname{mes}\left(Q^{s}\right)\right|=C M_{1}+M_{1}^{2}=M_{2},
\end{aligned}
$$

where $M_{2}=C M_{1}+M_{1}^{2}$ and $M_{1}$ is independent of both the random variables $\omega^{s}$ and the local coordinate $\xi$. Therefore, for the random variable $\omega$ in Section 2, $\left|\hat{a}_{i j h k}^{h_{0}}(\omega)\right|<M_{2}$.

Lemma 3.2. If $\omega$ is a random variable and $\hat{a}_{i j h k}^{h_{0}}(\omega)$ are defined as above, then one unique expected value of the equivalent mechanical parameters tensor $E_{\omega} \widehat{a}_{i j h k}^{h_{0}}(\omega)$ exists in the probability space $P=$ $\left(P_{a}\right)^{N} \times\left(P_{b}\right)^{N} \times\left(P_{b}\right)^{N} \times\left(P_{\theta_{a x_{1} x_{2}}}\right)^{N} \times \cdots \times\left(P_{x_{0}}\right)^{N} \times\left(P_{y_{0}}\right)^{N} \times\left(P_{z_{0}}\right)^{N}$.

Proof. From the definition of the long axis $a$, the middle axis $b$, the short axis $c$, the directions of the long axis and the middle axis, $\theta_{a_{x_{1} x_{2}}}, \theta_{a_{x_{1}}}$, and $\theta_{b_{x_{1} x_{2}}}, \theta_{b_{x_{1}}}$, and the coordinates of the central points of the ellipsoids $\left(x_{1^{0}}, x_{2^{0}}, x_{3^{0}}\right)$, their probability density functions are denoted by $f_{a}(x), f_{b}(x), f_{c}(x), f_{\theta_{a_{x_{1} x_{2}}}}(x), f_{\theta_{a_{x_{1}}}}(x), f_{\theta_{b_{x_{1} x_{2}}}}(x)$, $f_{\theta_{b_{1}}}(x), f_{x_{10}}(x), f_{x_{20}}(x)$, and $f_{x_{30}}(x)$, respectively. The united random variable $\omega=$ $\left(a_{1}, b_{1}, c_{1}, \theta_{a_{x_{1} x_{2} 1}}, \theta_{a_{x_{11}}}, \theta_{b_{x_{1} x_{21}}}, \theta_{b_{x_{1}}}, x_{1^{0} 1}, x_{2^{0} 1}, x_{3^{0} 1} \ldots, \theta_{b_{x_{1}}}, x_{1^{0} N}, x_{2^{0} N}, x_{3^{0} N}\right)$ has the the united probability density function $f_{a_{1}, b_{1}, c_{1}, \ldots, x_{0 N}, y_{0_{N}}, z_{0 N}}(\omega)=f_{a}^{N}(\omega) \cdot f_{b}^{N}(\omega) \cdot f_{c}^{N}(\omega) \cdots f_{x_{0}}^{N}(\omega) \cdot f_{y_{0}}^{N}(\omega)$. $f_{z_{0}}^{N}(\omega)$. From Lemma 3.1, one can obtain

$$
\begin{aligned}
E_{\omega} \widehat{a}_{i j h k}^{h_{0}}(\omega) & =\int_{P} \hat{a}_{i j h k}^{h_{0}}(\omega) \mathbf{d} P=\int_{-\infty}^{+\infty} \cdots \int_{-\infty}^{+\infty} \hat{a}_{i j h k}^{h_{0}}(\omega) f_{a_{1}, b_{1}, c_{1}, \ldots, x_{0 N}, y_{0_{N}}, z_{0 N}}(\omega) \mathbf{d} \omega \\
& <M_{2} \int_{-\infty}^{+\infty} \cdots \int_{-\infty}^{+\infty} f_{a_{1}, b_{1}, c_{1}, \ldots, x_{0 N}, y_{0_{N}}, z_{0 N}}(\omega) \mathbf{d} \omega \\
& <M_{2} \int_{-\infty}^{+\infty} \cdots \int_{-\infty}^{+\infty} f_{a}^{N}(\omega) \mathbf{d} \omega \cdots \int_{-\infty}^{+\infty} \cdots \int_{-\infty}^{+\infty} f_{z_{0}}^{N}(\omega) \mathbf{d} \omega \\
& <M_{2} .
\end{aligned}
$$

Therefore, there exists one unique expected value of the equivalent mechanical parameters tensor $E_{\omega} \widehat{a}_{i j h k}^{h_{0}}(\omega)(i, j, h, k=1,2, \ldots, n)$ in the probability space $P$. 
Lemma 3.3. If $\hat{a}_{i j h k}^{h_{0}}\left(\omega^{s}\right)(i, j, h, k=1,2, \ldots, n . s=1,2, \ldots)$ have the expected value $E_{\omega} \hat{a}_{i j h k}^{h_{0}}(\omega)$ in the probability space and $\omega$ is the random variable, one obtain

$$
\frac{\sum_{s=1}^{M} \widehat{a}_{i j h k}^{h_{0}}\left(\omega^{s}\right)}{M} \stackrel{a \cdot e}{\rightarrow} E_{\omega} \hat{a}_{i j h k}^{h_{0}}\left(\omega^{s}\right) \quad(M \longrightarrow \infty) .
$$

Proof. Because $\left\{\hat{a}_{i j h k}^{h_{0}}\left(\omega^{s}\right), s \geqslant 1\right\}$ are the independent and identical distribution random variables and

$$
S_{M}=\sum_{s=1}^{M} \widehat{a}_{i j h k}^{h_{0}}\left(\omega^{s}\right) \quad(M=1,2, \ldots)
$$

from Lemma 3.2, $\left|E_{\omega} \widehat{a}_{i j h k}^{h_{0}}\left(\omega^{s}\right)\right|<\infty$. Set $a_{1}=E_{\omega} \widehat{a}_{i j h k}^{h_{0}}(\omega)$; from Kolmogorov's classical strong law of large numbers, one obtains

$$
\frac{S_{M}}{M}-a_{1} \stackrel{a \cdot e}{\rightarrow} 0 \quad(M \longrightarrow \infty) .
$$

Therefore, we have $S_{M} / M \stackrel{a \cdot e}{\rightarrow} E_{\omega} \widehat{a}_{i j h k}^{h_{0}}\left(\omega^{s}\right)(M \longrightarrow \infty)$, that is,

$$
\frac{\sum_{s=1}^{M} \widehat{a}_{i j h k}^{h_{0}}\left(\omega^{s}\right)}{M} \stackrel{a \cdot e}{\rightarrow} E_{\omega} \widehat{a}_{i j h k}^{h_{0}}(\omega) \quad(M \longrightarrow \infty)
$$

Theorem 3.4. If $\widehat{a}_{i j h k}^{r, h_{0}}(\omega)(r=m, m-1, \ldots, 1)$ are computed as the equivalent mechanical parameter tensor of the composite material with $r$-scale random grains by the SMSA-FE algorithm, then the expected values of the equivalent mechanical parameters tensor $E_{\omega} \widehat{a}_{i j h k}^{r, h_{0}}(\omega)$ exist in the probability space $P=\left(P_{a}^{r}\right)^{N} \times\left(P_{b}^{r}\right)^{N} \times\left(P_{c}^{r}\right)^{N} \times \cdots \times\left(P_{x_{0}}^{r}\right)^{N} \times\left(P_{y_{0}}^{r}\right)^{N} \times\left(P_{z_{0}}^{r}\right)^{N}$.

Proof. Set $r=m$; define

$$
a_{i j h k}\left(\frac{x}{\varepsilon^{r}}, \omega^{s}\right)= \begin{cases}a_{i j h k^{\prime}}^{2}, & x \in \varepsilon \widehat{Q}^{s}, \\ a_{i j h k^{\prime}}^{1}, & x \in \varepsilon \widetilde{Q}^{s},\end{cases}
$$

because both $a_{i j h k}^{1}$ and $a_{i j h k}^{2}$ are constants satisfying $\left|\max \left\{a_{i j h k}^{1}, a_{i j h k}^{2}\right\}\right|<M_{1}$. It is easy to see that $a_{i j h k}\left(x / \varepsilon^{r}, \omega^{s}\right)(i, j=1,2,3)$ are bounded and measurable random variables. Based on Lemmas 3.1, 3.2, and 3.3, there exist the expected values $E_{\omega} \widehat{a}_{i j h k}^{r, h_{0}}(\omega)$ of the equivalent mechanical parameter tensor $\hat{a}_{i j h k}^{r, h_{0}}(\omega)$ of the material with only the $r$ th random grains. Therefore, the equivalent mechanical parameter tensor of the composites with the $r$ th random grains can exist as follows:

$$
\frac{\sum_{s=1}^{M} \widehat{a}_{i j h k}^{r, h_{0}}\left(\omega^{s}\right)}{M} \stackrel{a \cdot e}{\rightarrow} E_{\omega} \widehat{a}_{i j h k}^{r, h_{0}}\left(\omega^{s}\right) \quad(M \longrightarrow \infty)
$$


Set $r=r-1$ and $\widehat{a}_{i j h k}^{r, h_{0}}\left(x / \varepsilon^{(r+1)}, \omega^{s}\right)=E_{\omega} \widehat{a}_{i j h k}^{r, h_{0}}\left(\omega_{r}\right)$, the mechanical parameters of the equivalent matrix material and the grain material in the equivalent composite material with the $r$ th random grains can be obtained.

That is,

$$
a_{i j h k}\left(\frac{x}{\varepsilon^{r}}, \omega^{s}\right)= \begin{cases}\hat{a}_{i j h k}^{r, h_{0}}\left(\frac{x}{\varepsilon^{(r+1)}}, \omega^{s}\right), & x \in \varepsilon \widehat{Q}^{s}, \\ a_{i j h k^{\prime}}^{1} & x \in \varepsilon \widetilde{Q}^{s},\end{cases}
$$

$\left|a_{i j h k}\left(x / \varepsilon^{r}, \omega^{s}\right)\right|<M_{1}$. It is easy to see that $a_{i j h k}\left(x / \varepsilon^{r}, \omega^{s}\right)(i, j=1,2,3)$ are bounded and measurable random variables. By the iterated loop proof for $r$ as above, based on Lemmas 3.2 and 3.3, the convergence of the equivalent mechanical parameter tensor $E_{\omega} \widehat{a}_{i j h k^{\prime}}^{r, h_{0}}$ $(r=m, m-1, \ldots, 1)$ can be obtained.

\section{Error Analysis for Equivalent Mechanical Parameter Tensor Computed by SMSA-FE Algorithm}

Based on the SMSA-FE algorithm, if the equivalent mechanical parameter tensor is computed, three kinds of errors are considered: the homogenization error, the random error based on Monte Carlo simulation method, and the finite element computation error. For the homogenization error, the composite materials with multiscale random grains are the special cases of the random coefficient problems whose convergence was proved in [14]. For the random error, we have obtained the convergence of the equivalent mechanical parameter tensor of the composite material with multiscale random grains as above. Therefore, in the following section, we will devote to analyzing the finite element error based on SMSA.

Firstly, we give the finite element error estimation of the statistical two-scale analysis. Then we give the error estimation of the SMSA-FE algorithm.

Lemma 4.1. If $N_{\alpha m}\left(\xi, \omega^{s}\right), \alpha=1,2, \ldots n$, is the variational solution of (2.6) and $N_{\alpha m}^{h_{0}}\left(\xi, \omega^{s}\right)$ is the corresponding finite element solution of $(2.6)$ and $N_{\alpha m}^{h_{0}}\left(\xi, \omega^{s}\right) \in\left(H^{2}(Q)\right)^{n}$, then we have

$$
\begin{gathered}
\left\|N_{\alpha m}\left(\xi, \omega^{s}\right)-N_{\alpha m}^{h_{0}}\left(\xi, \omega^{s}\right)\right\|_{\left(H_{0}^{1}(Q)\right)^{n}} \leq C h_{0}\left\|N_{\alpha m}\left(\xi, \omega^{s}\right)\right\|_{\left(H^{2}(Q)\right)^{n}}, \\
\left\|N_{\alpha m}\left(\xi, \omega^{s}\right)-N_{\alpha m}^{h_{0}}\left(\xi, \omega^{s}\right)\right\|_{\left(L^{2}(Q)\right)^{n}} \leq C h_{0}^{2}\left\|N_{\alpha m}\left(\xi, \omega^{s}\right)\right\|_{\left(H^{2}(Q)\right)^{n}},
\end{gathered}
$$

where the constant $C>0$ is independent of the size $h_{0}$ of mesh.

Proof. Since $E_{\alpha m}^{s}=\mathbf{N}_{\alpha m}\left(\xi, \omega^{s}\right)-\mathbf{N}_{\alpha m}^{h_{0}}\left(\xi, \omega^{s}\right) \in H_{0}^{1}(Q)$, based on Korn inequality and the interpolation theorem, we have that 


$$
\begin{aligned}
\left\|E_{\alpha m}^{S}\right\|_{\left(H^{1}(Q)\right)^{n}}^{2} & \leq C a\left(E_{\alpha m}^{s}, E_{\alpha m}^{S}\right) \\
& =C a\left(\mathbf{N}_{\alpha m}\left(\xi, \omega^{s}\right)-\mathbf{N}_{\alpha m}^{h_{0}}\left(\xi, \omega^{s}\right), \mathbf{N}_{\alpha m}\left(\xi, \omega^{s}\right)-\gamma_{h_{0}} \mathbf{N}_{\alpha m}^{h_{0}}\left(\xi, \omega^{s}\right)\right) \\
& \leq C h_{0}\left\|\mathbf{N}_{\alpha m}\left(\xi, \omega^{s}\right)\right\|_{\left(H^{2}(Q)\right)^{n}}\left\|E_{\alpha m}^{s}\right\|_{\left(H^{1}(Q)\right)^{n}} .
\end{aligned}
$$

That is,

$$
\left\|N_{\alpha m}\left(\xi, \omega^{s}\right)-N_{\alpha m}^{h_{0}}\left(\xi, \omega^{s}\right)\right\|_{\left(H^{1}(Q)\right)^{n}} \leq C h_{0}\left\|N_{\alpha m}\left(\xi, \omega^{s}\right)\right\|_{\left(H^{2}(Q)\right)^{n}} .
$$

Aubin-Nitsche lemma [28] yields

$$
\left\|N_{\alpha m}\left(\xi, \omega^{s}\right)-N_{\alpha m}^{h_{0}}\left(\xi, \omega^{s}\right)\right\|_{\left(L^{2}(Q)\right)^{n}} \leq C h_{0}{ }^{2}\left\|N_{\alpha m}\left(\xi, \omega^{s}\right)\right\|_{\left(H^{2}(Q)\right)^{n}} .
$$

Lemma 4.2. Let $N_{\alpha p m}\left(\xi, \omega^{s}\right), \alpha_{1}=1,2, \ldots, n$, be the variational solution of the (2.6) and $N_{\alpha p m}^{h_{0}}\left(\xi, \omega^{s}\right)$ the finite element solution such that $N_{\alpha p m}\left(\xi, \omega^{s}\right) \in W^{2, \infty}(Q)$, and then one has

$$
\begin{gathered}
\left\|\nabla_{\xi}\left(N_{\alpha p m}\left(\xi, \omega^{s}\right)-N_{\alpha p m}^{h_{0}}\left(\xi, \omega^{s}\right)\right)\right\| \leq C h_{0}\left|\ln h_{0}\right|\left\|\nabla_{\xi}^{2} N_{\alpha p m}\right\|_{L^{\infty}(Q)}, \\
\left\|\left(N_{\alpha p m}\left(\xi, \omega^{s}\right)-N_{\alpha p m}^{h_{0}}\left(\xi, \omega^{s}\right)\right)\right\| \leq C h_{0}{ }^{2}\left|\ln h_{0}\right|\left\|\nabla_{\xi}^{2} N_{\alpha p m}\right\|_{L^{\infty}(Q)} .
\end{gathered}
$$

In fact, based on the idea and the method in [28], it is easy to prove Lemma 4.2.

Lemma 4.3. Let $\widehat{a}_{i j h k}$ be the equivalent mechanical parameter tensor matrix based on STSA and $\widehat{a}_{i j h k}^{h_{0}}$ its finite element approximation as above, if there exists one constant $\widehat{N}$ such that for all $\omega^{s} \in$ $P,\left\|N_{\alpha p m}\left(\xi, \omega^{s}\right)\right\|_{H^{2}(Q)} \leq \widehat{N}$. Then one has

$$
\left\|\widehat{a}_{i j h k}-\widehat{a}_{i j h k}^{h_{0}}\right\|_{L^{\infty}} \leq C h_{0}\left|\ln h_{0}\right| \widehat{N}
$$

Proof. From the above algorithm, the following equation is held.

$$
\widehat{a}_{i j h k}=\widehat{a}_{i j h k}^{h_{0}}+R_{i j h k}
$$

where $R_{i j h k}$ is defined by

$$
R_{i j h k}=\frac{\sum_{s=1}^{M} \int_{Q} a_{i p h q}\left(\xi, \omega^{s}\right)(1 / 2)\left(\mathscr{H} / \partial \xi_{p}+\mathcal{A} / \partial \xi_{q}\right)}{M}
$$

where $\mathscr{\ell}$ denotes $\partial\left(N_{k q j}\left(\xi, \omega^{s}\right)-N_{k q j}^{h_{0}}\left(\xi, \omega^{s}\right)\right)$ and $\mathscr{A}$ denotes $\partial\left(N_{k p j}\left(\xi, \omega^{s}\right)-N_{k p j}^{h_{0}}\left(\xi, \omega^{s}\right)\right)$ 
Since $\|R\|_{\infty}$ denote the maximum norm of matrix $\left(R_{i j h k}\right)_{n \times n}$, applying Lemma 4.2 to the above (4.8), we deduce

$$
\begin{aligned}
\|R\|_{\infty} & =\left\|\frac{\sum_{s=1}^{M} \int_{Q} a_{i p h q}\left(\xi, \omega^{s}\right)(1 / 2)\left(\mathscr{\ell} / \partial \xi_{p}+\mathcal{A} / \partial \xi_{q}\right)}{M}\right\|_{\infty} \\
& \leq \frac{1}{M} \sum_{s=1}^{M}\left\|\int_{Q} a_{i p h q}\left(\xi, \omega^{s}\right) \frac{1}{2}\left(\frac{\mathscr{\ell}}{\partial \xi_{p}}+\frac{\mathcal{A}}{\partial \xi_{q}}\right)\right\|_{\infty} \\
& \leq C \frac{1}{M} h_{0}\left|\ln h_{0}\right| \sum_{s=1}^{M}\left\|\nabla_{\xi}^{2} N_{k}\left(\xi, \omega^{s}\right)\right\|_{L^{\infty}}
\end{aligned}
$$

Since there exists one constant $\widehat{N}$ such that the function matrices $\left\|N_{\alpha}\left(\xi, \omega^{s}\right)\right\| \leq \widehat{N}$, the above inequality (4.9) yields

$$
\|R\|_{\infty} \leq C h_{0}\left|\ln h_{0} \| \widehat{N}\right|
$$

Then inequality (4.6) follows from the above inequality (4.10).

Based on the SMSA-FE algorithm, the equivalent mechanical parameter tensor of the equivalent material with the $m$ th random grains can be obtained. Therefore, the matrix material with $(m-1)$ th random grains can be considered as the equivalent matrix material. Using the loop proof by Lemmas 4.2 and 4.3, it is easy to obtain the following theorem on the equivalent mechanical parameter tensor of a composite material with $m$-scale random grains.

Theorem 4.4. Let $\widehat{a}_{i j h k}^{r}(r=m, m-1, \ldots, 1)$ be the equivalent mechanical parameters tensor of the composite material with $r$ th scale random grains and $\hat{a}_{i j h k}^{r, h_{0}}(r=m, m-1, \ldots, 1)$ its finite element approximation; set the size of the last mesh of the finite element in $Q^{s}$ to be $h_{0}$; based on the SMSA-FE algorithm, if there exists one constant $\widehat{N}$ such that for all, $\omega^{s} \in P,\left\|N_{\alpha p m}\left(\xi_{r}, \omega^{s}\right)\right\|_{H^{2}(Q)} \leq \widehat{N},(r=$ $m, m-1, \ldots, 1)$, then one has

$$
\left\|\widehat{a}_{i j h k}^{r}-\widehat{a}_{i j h k}^{r, h_{0}}\right\| \leq C h_{0}\left|\ln h_{0}\right| \widehat{N}
$$

where $C$ is one constant that is independent of $h_{0}$ but dependent on the sizes of the other finite element mesh $h_{0 r}(r=m, m-1, \ldots, 2)$ in the cell $Q_{r}(r=m, m-1, \ldots, 2)$ with rth random grains.

From Theorem 4.4, it is easy to see the error of the equivalent mechanical parameter tensor of the composite material with the biggest grains being the main error by the SMSAFE algorithm. Hence, we only need to consider the error in the composite material with the biggest random grains. 


\section{Symmetry and Positive Definite Property for Equivalent Mechanical Parameter Tensor}

From $[7,26]$, if the parameters of the ellipsoids are subjected to the uniform probability $P$, the equivalent mechanical parameter tensor $\widehat{a}_{i j h k}$ shall satisfy the following conditions:

$$
\begin{gathered}
\widehat{a}_{i j h k}=\widehat{a}_{j i k h}=\widehat{a}_{h j i k,} \\
\mu_{1} \eta_{i j} \eta_{i j} \leq \widehat{a}_{i j h k} \eta_{i j} \eta_{h k} \leq \mu_{2} \eta_{i j} \eta_{i j},
\end{gathered}
$$

where for any symmetry matrix $\eta=\left(\eta_{i j}\right)_{n \times n}, \mu_{1} \geq 0$, and $\mu_{2} \geq 0$.

Therefore, if the parameters of the ellipsoids are subjected to the uniform probability $P$, it is important to keep the symmetric, positive definite property of mechanical parameter tensor computed by the finite element method. So we shall give the following theorem to illustrate it.

Lemma 5.1. Let $\left\{\widehat{a}_{i j h k}\right\}$ be the equivalent mechanical parameter tensor based on STSA [26] and $\hat{a}_{i j h k}^{h_{0}}$ its finite element approximation; if there exists one constant $\widehat{N}$ such that for all $\omega^{s} \in$ $P,\left\|N_{\alpha p m}\left(\xi, \omega^{s}\right)\right\|_{H^{2}(Q)} \leq \widehat{N}$, the matrix $\widehat{a}_{i j h k}^{h_{0}}$ satisfies the following property:

$$
\begin{gathered}
\hat{a}_{i j h k}^{h_{0}}=\widehat{a}_{j i k h}^{h_{0}}=\widehat{a}_{h j i k^{\prime}}^{h_{0}} \\
\widehat{K}_{1} \eta_{i j} \eta_{i j} \leq \widehat{a}_{i j h k}^{h_{0}} \eta_{i j} \eta_{h k} \leq \widehat{K}_{2} \eta_{i j} \eta_{i j}
\end{gathered}
$$

for any symmetric matrix $\eta=\left(\eta_{i h}\right)_{n \times n}$, where $\widehat{K}_{1}, \widehat{K}_{2}$ are positive constants.

Proof. Taking into consideration the fact that $\widehat{a}_{i j h k}\left(\omega^{s}\right)=\widehat{a}_{j i k h}\left(1^{s}\right)=\widehat{a}_{h j i k}\left(\omega^{s}\right)$, based on the concept of $N_{\alpha}^{h_{0}}\left(\xi, \omega^{s}\right)$, taking into account the idea in [7], we deduce that

$$
\begin{aligned}
\hat{a}_{j i k h}^{h_{0}}\left(\omega^{s}\right) & =\frac{1}{\left|Q^{s}\right|} \int_{Q^{s}}\left[a_{j i k h}\left(\xi, \omega^{s}\right)+a_{j p k q}\left(\xi, \omega^{s}\right) \frac{1}{2}\left(\frac{\partial N_{h p i}^{h_{0}}\left(\xi, \omega^{s}\right)}{\xi_{q}}+\frac{\partial N_{h q i}^{h_{0}}\left(\xi, \omega^{s}\right)}{\xi p}\right)\right] \mathrm{d} \xi \\
& =\frac{1}{\left|Q^{s}\right|} \int_{Q^{s}}\left[a_{h j i k}\left(\xi, \omega^{s}\right)+a_{h q i p}\left(\xi, \omega^{s}\right) \frac{1}{2}\left(\frac{\partial N_{k p j}^{h_{0}}\left(\xi, \omega^{s}\right)}{\xi_{q}}+\frac{\partial N_{k q j}^{h_{0}}\left(\xi, \omega^{s}\right)}{\xi_{p}}\right)\right] \mathrm{d} \xi \\
& =\widehat{a}_{k j i h}^{h_{0}}\left(\omega^{s}\right) .
\end{aligned}
$$

So we have proved $\hat{a}_{j i k h}^{h_{0}}\left(\omega^{s}\right)=\widehat{a}_{h j i k}^{h_{0}}\left(\omega^{s}\right)$. Let us establish the first equality in (5.2), which is equivalent to prove $\left(\widehat{A}^{h_{0} h k}\right)^{*}=\widehat{A}^{h_{0} k h}$, where $A^{*}$ denotes the transpose of the matrix $A$ and $\widehat{A}^{h_{0} h k}$ is the matrix $\left(\widehat{a}_{i j h k}^{h_{0}}\right)_{n \times n}$. 
From the integral identity for solution of problem (2.6), for any matrix $M^{h_{0}}(\xi) \in$ $\left(V^{h_{0}}\left(Q^{s}\right)\right)^{n \times n}$, we have

$$
-\int_{Q^{s}} \frac{\partial(M(\xi)}{\partial \xi_{p}} A^{p q}\left(\xi, \omega^{s}\right) \frac{\partial\left(N_{k}^{*}\left(\xi, \omega^{s}\right)\right)}{\partial \xi_{q}} d \xi=\int_{Q^{s}} A^{p k}\left(\xi, \omega^{s}\right) \frac{(M(\xi))}{\partial \xi_{p}} d \xi
$$

Based on the relations $\left(A^{p q}(\xi)\right)^{*}=A^{q p}(\xi)$ and $(A B)^{*}=B^{*} A^{*}$ for matrices $A, B$, it is easy to obtain $N_{\alpha}$ from (5.5) that

$$
-\int_{Q^{s}} \frac{\partial N_{k}^{h_{0}}\left(\xi, \omega^{s}\right)}{\partial \xi_{p}} A^{q p}\left(\xi, \omega^{s}\right) \frac{\partial M^{*}(\xi)}{\partial \xi_{q}} d \xi=\int_{Q^{s}} A^{k q}\left(\xi, \omega^{s}\right) \frac{\partial M^{*}(\xi)}{\partial \xi_{q}} d \xi .
$$

If we set $M=N_{h}^{h_{0} \star}$, the following equations are obtained.

$$
-\int_{Q^{s}} \frac{\partial N_{k}^{h_{0}}\left(\xi, \omega^{s}\right)}{\partial \xi_{p}} A^{p q}\left(\xi, \omega^{s}\right) \frac{\partial N_{h}^{h_{0}}\left(\xi, \omega^{s}\right)}{\partial \xi_{q}} d \xi=\int_{Q^{s}} A^{k q}\left(\xi, \omega^{s}\right) \frac{\partial N_{h}^{h_{0}}\left(\xi, \omega^{s}\right)}{\partial \xi_{q}} d \xi .
$$

Taking into account the idea in [7], (5.7), and the concept of $N_{h}^{h_{0} *}\left(\xi, \omega^{s}\right)$, the following equations can be obtained.

$$
\begin{aligned}
\int_{Q^{s}} \frac{\partial}{\partial \xi_{q}}\left(N_{k}^{h_{0}^{*}}+\xi_{k} E\right) A^{q p}\left(\xi, \omega^{s}\right) \frac{\partial}{\partial \xi_{p}}\left(N_{h}^{h_{0}}+\xi h E\right) d \xi \\
=\int_{Q^{s}}\left(\frac{\partial N_{k}^{h_{0} *}}{\partial \xi_{q}} A^{q p}\left(\xi, \omega^{s}\right) \frac{\partial N_{h}^{h_{0}}}{\partial \xi_{p}}+\delta_{q k} A^{q p}\left(\xi, \omega^{s}\right) \frac{\partial N_{h}^{h_{0}}}{\partial \xi_{p}}+\frac{\partial N_{k}^{h_{0} *}}{\partial \xi_{q}} A^{q p}\left(\xi, \omega^{s}\right) \delta_{p h} E\right. \\
\left.\quad+\delta_{q k} A^{q p}\left(\xi, \omega^{s}\right) \delta_{p h} E\right) d \xi \\
=\int_{Q^{s}}\left(\frac{\partial N_{k}^{h_{0} *}}{\partial \xi_{q}} A^{q p}\left(\xi, \omega^{s}\right) \frac{\partial N_{h}^{h_{0}}}{\partial \xi_{p}}+A^{k p}\left(\xi, \omega^{s}\right) \frac{\partial N_{h}^{h_{0}}}{\partial \xi_{p}}+\frac{\partial N_{k}^{h_{0} *}}{\partial \xi_{q}} A^{q h}\left(\xi, \omega^{s}\right)+A^{k h}\left(\xi, \omega^{s}\right)\right) d \xi \\
=\int_{Q^{s}}\left(\frac{\partial N_{k}^{h_{0} *}}{\partial \xi_{q}} A^{q h}\left(\xi, \omega^{s}\right)+A^{k h}\left(\xi, \omega^{s}\right) d \xi\right)=\left(A^{h_{0} h k \star}\right)\left(\omega^{s}\right) .
\end{aligned}
$$

In the second equation of the above equations, the property of $\delta_{i j}$ is used; in the third equation, (5.7) is applied. It follows that the equivalent mechanical parameter matrices can be written in the following form by the relationship of $A^{j{ }^{\star}}=A^{q j}$ :

$$
\begin{aligned}
& \widehat{A}^{h_{0} h k}\left(\omega^{s}\right)=\int_{Q} \frac{\partial}{\partial \xi_{q}}\left(N_{h}^{h_{0} *}+\xi_{h} E\right) A^{p q}\left(\xi, \omega^{s}\right) \frac{\partial}{\partial \xi_{p}}\left(N_{k}^{h_{0}}+\xi_{k} E\right) d \xi \\
& \widehat{A}^{h_{0} k h}\left(\omega^{s}\right)=\int_{Q^{s}} \frac{\partial}{\partial \xi_{q}}\left(N_{k}^{h_{0} *}+\xi_{k} E\right) A^{q j}\left(\xi, \omega^{s}\right) \frac{\partial}{\partial \xi_{j}}\left(N_{h}^{h_{0}}+\xi_{h} E\right) d \xi
\end{aligned}
$$


Thus

$$
\left(\widehat{A}^{h_{0} k h}\right)^{*}\left(\omega^{s}\right)==\int_{Q^{s}} \frac{\partial}{\partial \xi_{j}}\left(N_{h}^{h_{0} *}+\xi_{h} E\right) A^{j q}\left(\xi, \omega^{s}\right) \frac{\partial}{\partial \xi_{q}}\left(N_{k}^{h_{0}}+\xi_{k} E\right) d \xi=\left(\widehat{A}^{h_{0} h k}\right)\left(\omega^{s}\right) .
$$

Hence, we see that $\widehat{A}^{h_{0} h k}\left(\omega^{s}\right)=\left(\widehat{A}^{h_{0} k h}\left(\omega^{s}\right)\right)^{*}$. Thus we obtain the following equations:

$$
\widehat{a}_{i j h k}^{h_{0}}\left(\omega^{s}\right)=\widehat{a}_{j i k h}^{h_{0}}\left(\omega^{s}\right)=\widehat{a}_{h j i k}^{h_{0}}\left(\omega^{s}\right) .
$$

Making use of the relations (5.11), one has

$$
\frac{1}{M} \sum_{s=1}^{M} \widehat{a}_{i j h k}^{h_{0}}\left(\omega^{s}\right)=\frac{1}{M} \sum_{s=1}^{M} \hat{a}_{j i k h}^{h_{0}}\left(\omega^{s}\right)=\frac{1}{M} \sum_{s=1}^{M} \widehat{a}_{h j i k}^{h_{0}}\left(\omega^{s}\right) .
$$

That is (5.2) is proved.

In the sequel, we shall prove (5.3). From the inequality (4.6) and Theorem 4.4, there exists one $h_{0} \geq 0$ that is small enough such that

$$
C h_{0}\left|\ln h_{0}\right|\left|\widehat{N}_{\alpha}\right|_{L^{\infty}}<\frac{\mu_{1}}{2}
$$

We have

$$
\frac{\mu_{1}}{2} \eta_{i j} \eta_{i j} \leq \widehat{a}_{i j h k}^{h_{0}} \eta_{i j} \eta_{h k} \leq\left(\mu_{2}+\frac{\mu_{1}}{2}\right) \eta_{i j} \eta_{i j}
$$

Setting $\widehat{K}_{1}=\mu_{1} / 2$ and $\widehat{K}_{2}=\mu_{2}+\mu_{1} / 2$ yields the inequality (5.3).

By the iterated multiscale analysis and Lemma 5.1, the finite element approximation of the equivalent mechanical parameter tensor of the composite material with multiscale random grains satisfies the following property.

Theorem 5.2. Let $\left\{\hat{a}_{i j h k}^{r}\right\}$ be the equivalent mechanics parameter tensor of the composite material with $r$-scale random grains based on the SMSA algorithm and $\hat{a}_{i j h k}^{r, h_{0}}$ their finite element approximation by (2.3). Set the size of the last mesh of the finite element in $Q^{s}$ to be $h_{0}$; if there exists the constant $\widehat{N}_{\alpha_{1}}$ such that for all, $\omega^{s} \in P,\left\|N_{\alpha p m}^{r}\left(\xi, \omega^{s}\right)\right\|_{H^{2}(Q)} \leq \widehat{N}_{\alpha p m}$, then $\widehat{a}_{i j h k}^{r, h_{0}}$ satisfy the conditions

$$
\begin{gathered}
\hat{a}_{i j h k}^{r, h_{0}}=\widehat{a}_{j i k h}^{r, h_{0}}=\widehat{a}_{h j i k^{\prime}}^{r, h_{0}} \\
\overline{K_{1}} \eta_{i j} \eta_{i j} \leq \widehat{a}_{i j h k}^{r, h_{0}} \eta_{i j} \eta_{h k} \leq \overline{K_{2}} \eta_{i j} \eta_{i j},
\end{gathered}
$$

where $\eta=\left(\eta_{i h}\right)_{n \times n}$ is the symmetry matrix and $\overline{K_{1}}, \overline{K_{2}}$ are the positive constants that are independent of $h_{0}$ but dependent on the sizes of the other finite element meshes $h_{0 r},(r=m, m-1, \ldots, 2)$ in the $r$ th scale cells $Q_{r}(r=m, m-1, \ldots, 2,1)$. 
Table 1: Probability distributions of grains in composite material.

\begin{tabular}{lccc}
\hline & Small grains & \multicolumn{2}{c}{ Large grains } \\
\hline$\theta$ & {$[0,2 \pi]$} & $\theta$ & {$[0,2 \pi]$} \\
$a$ & {$[0.03,0.08]$} & $a$ & {$[0.1,1]$} \\
$b$ & {$[0.02, a]$} & $b$ & {$[0.1, a]$} \\
\hline
\end{tabular}
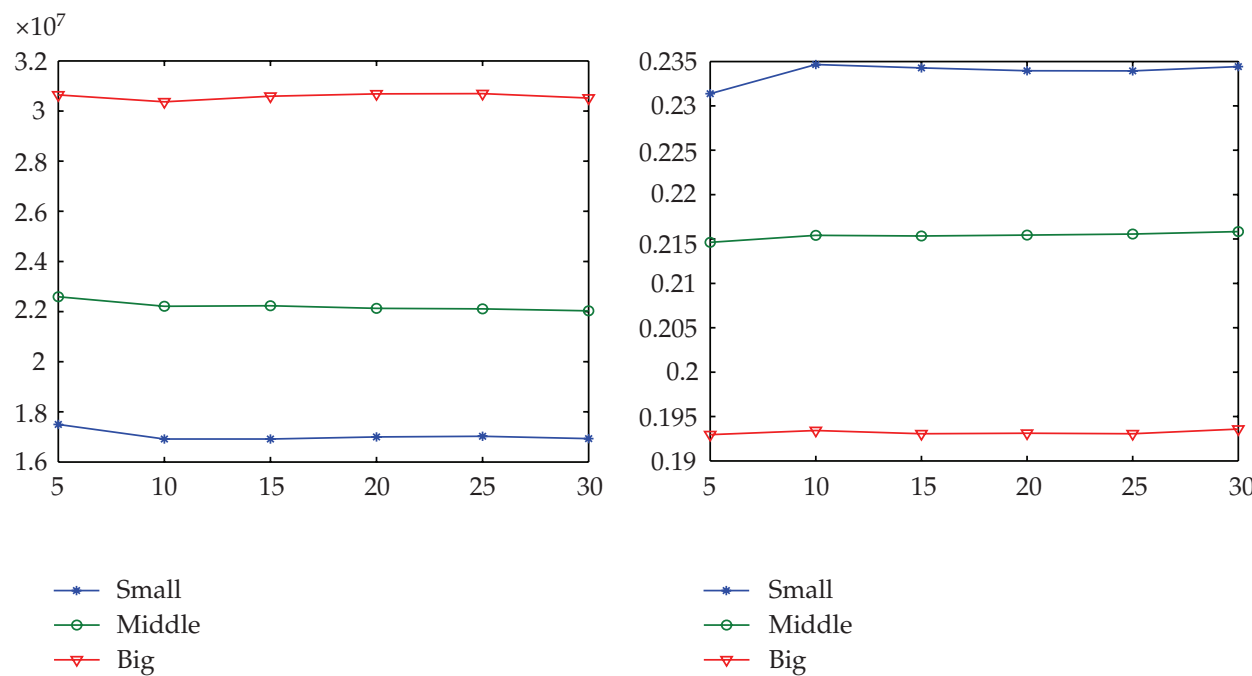

(a)

(b)

Figure 2: Young's modulus and Poisson's ratio for 5, 10, 15,.., 30 samples with different scale random grains by the SMSA-FE procedure.

\section{Numerical Experiment}

To test the validity of the error result, the convergence, and the symmetric, positive definite property of the mechanical parameter tensor computed by the SMSA-FE algorithm, two numerical examples are given as follows.

The first example models a composite material. The grains are divided into two classes according to the sizes of their long axis shown in Table 1 . We use one statistical window $\varepsilon^{1}=0.1$ to predict the mechanical parameters of the equivalent matrix with small random grains. In each window, small grains occupy approximate $30 \%$ of the volume. Their long axis $a$, short axis $b$, and angle $\theta$ are subjected to the uniform distributions shown in Table 1 . Using the different finite element sizes $h_{0}$, based on the data of Table 2 , we obtain the equivalent mechanical parameters tensor $\left\{\widehat{a}_{i j h k}\right\}$ that are given in Table 3 .

From Table 3, it is easy to see that convergence of the equivalent mechanical parameter tensor computed by the SMSA-FE algorithm exists. From Table 3, the symmetric, positive definite property of the equivalent mechanical parameter tensor and the convergence of the finite element errors with the different mesh sizes $h_{0}$ are proved.

The second example is a concrete named as C30 with three-scale random rock grains whose sizes are from $0.3 \mathrm{~mm}$ to $19 \mathrm{~mm}$. Its matrix is made up of the cement and the sand. Their sizes of the three-scale rock grains in the concrete are shown in Table 4. Their elastic parameters are shown in Table 5. If all grains are generated in a large statistical window of 
Table 2: Mechanical parameters of the matrix and grains.

\begin{tabular}{lccccc}
\hline & Matrix & \multicolumn{3}{c}{ Grains } \\
\hline $8.415 \times 10^{5}$ & $7.041 \times 10^{5}$ & 0 & $2.99 \times 10^{6}$ & $1.50 \times 10^{6}$ & 0 \\
$7.041 \times 10^{5}$ & $8.415 \times 10^{5}$ & 0 & $1.50 \times 10^{6}$ & $2.99 \times 10^{6}$ & 0 \\
0 & 0 & $6.870 \times 10^{4}$ & 0 & 0 & $7.50 \times 10^{5}$ \\
\hline
\end{tabular}

Table 3: Equivalent mechanical parameters $\left\{\widehat{a}_{i j}\left(\omega^{s}\right)\right\}$ for different mesh sizes $h_{0}$.

\begin{tabular}{lccccc}
\hline$h_{0}$. & $a_{11}$ & $a_{12}$ & $a_{21}$ & $a_{22}$ & $a_{33}$ \\
\hline 0.04 & $1.083 \times 10^{6}$ & $8.323 \times 10^{5}$ & $8.323 \times 10^{5}$ & $1.092 \times 10^{6}$ & $1.327 \times 10^{5}$ \\
0.02 & $1.095 \times 10^{6}$ & $8.302 \times 10^{5}$ & $8.302 \times 10^{5}$ & $1.099 \times 10^{6}$ & $1.295 \times 10^{5}$ \\
0.01 & $1.097 \times 10^{6}$ & $8.377 \times 10^{5}$ & $8.373 \times 10^{5}$ & $1.108 \times 10^{6}$ & $1.319 \times 10^{5}$ \\
0.005 & $1.098 \times 10^{6}$ & $8.375 \times 10^{5}$ & $8.371 \times 10^{5}$ & $1.112 \times 10^{6}$ & $1.311 \times 10^{5}$ \\
\hline
\end{tabular}

Table 4: Size of rock random grains and the number in a statistical window.

\begin{tabular}{lcccc}
\hline Class & Grain size range & Average size & Number of rock grains & Sizes of statistical windows \\
\hline Large grains & $10-19 \mathrm{~mm}$ & $14.5 \mathrm{~mm}$ & 8 & $\varepsilon^{1}=100 \mathrm{~mm}$ \\
Middle grains & $1-10 \mathrm{~mm}$ & $5 \mathrm{~mm}$ & 20 & $\varepsilon^{2}=50 \mathrm{~mm}$ \\
Small Grains & $0.3-1 \mathrm{~mm}$ & $0.6 \mathrm{~mm}$ & 28 & $\varepsilon^{2}=5 \mathrm{~mm}$ \\
\hline
\end{tabular}

Table 5: Elasticity mechanical parameters of the matrix, grains, and joint interface materials.

\begin{tabular}{lcc}
\hline Class & Young's modulus $(\mathrm{GPa})$ & Poisson's ratio \\
\hline Rock grains & 74.5 & 0.15 \\
Matrix & 13.5 & 0.25 \\
Joint interface & 50.5 & 0.20 \\
\hline
\end{tabular}

Table 6: Equivalent mechanical parameters of a composites with small rock random grains by the SMSA-FE algorithm (kPa).

\begin{tabular}{lccccc}
\hline 20341770.6 & 6054537.6 & 6054645.8 & -119951.6 & 164516.8 & -12764.7 \\
6054537.6 & 20128556.8 & 6060485.8 & -106579.5 & 19649.2 & -141765.4 \\
6054645.8 & 6060485.8 & 20061719.4 & -18765.7 & 140779.4 & -116731.9 \\
0.000000 & 0.000000 & 0.000000 & 6923128.0 & 0.000000 & 0.000000 \\
164516.8 & 19649.25 & 140779.48 & 0.000000 & 6902337.6 & -81061.0 \\
-12764.7 & -141765.4 & 0.000000 & 98172.3 & -81061.0 & 6859146.1 \\
\hline
\end{tabular}

Table 7: Equivalent mechanical parameter tensor for concrete with middle rock random grains by the SMSA-FE algorithm ( $\mathrm{kPa})$.

\begin{tabular}{lccccc}
\hline 25464900.2 & 6882577.0 & 6881924.2 & -135341.116667 & 111669.7 & -2238.3 \\
6882577.0 & 25410941.8 & 6916568.5 & -123921.9 & 7075.0 & -187899.58 \\
6881924.2 & 6916568.5 & 25291123.2 & -17145.9 & 89634.5 & -192916.5 \\
0.000000 & 0.000000 & 0.000000 & 9071093.3 & 0.000000 & 0.000000 \\
111669.7 & 7075.0 & 89634.5 & 0.000000 & 9042380.8 & -82547.1 \\
-2238.3 & -187899.5 & 0.000000 & 69773.7 & -82547.1 & 9060481.0 \\
\hline
\end{tabular}


Table 8: Equivalent mechanical parameter tensor for concrete with large rock random grains by the SMSA-FE algorithm ( $\mathrm{kPa})$.

\begin{tabular}{lccccc}
\hline 34510679.4 & 8073028.8 & 8076036.2 & -75074.2 & 111127.6 & -4769.6 \\
8073025.0 & 34121732.2 & 8092588.8 & -36814.0 & 5862.0 & -110837.9 \\
8076036.8 & 8092589.3 & 34065284.2 & -531.7 & 123664.7 & -79363.6 \\
0.000000 & 0.000000 & 0.000000 & 12860237.8 & 0.000000 & 0.000000 \\
111127.6 & 5862.0 & 123664.7 & 0.000000 & 12862877.8 & -30079.5 \\
-4769.6 & -110837.9 & 0.000000 & 78302.2 & -30079.5 & 12783465.6 \\
\hline
\end{tabular}

Table 9: Equivalent mechanical parameter tensor for concrete with large rock random grains by the SMSA-FE algorithm (GPa).

\begin{tabular}{lcccccc}
\hline Iterative number & 5 & 10 & 15 & 20 & 25 & 30 \\
\hline$E_{\text {small }}$ & 17.4985392 & 16.9160384 & 16.9151317 & 17.0004448 & 17.0245196 & 16.9342101 \\
$V_{\text {small }}$ & 0.231375 & 0.234653 & 0.234271 & 0.233951 & 0.233942 & 0.233926 \\
$E_{\text {middle }}$ & 22.5917136 & 22.2098256 & 22.2298752 & 22.1302736 & 22.1057408 & 22.0321386 \\
$V_{\text {middle }}$ & 0.214631 & 0.215408 & 0.215342 & 0.215443 & 0.215552 & 0.215537 \\
$E_{\text {large }}$ & 30.644806 .4 & 30.3689408 & 30.5923968 & 30.6815936 & 30.6936934 & 30.6862218 \\
$V_{\text {large }}$ & 0.192951 & 0.193419 & 0.193065 & 0.193116 & 0.193066 & 0.193082 \\
\hline
\end{tabular}

$500 \mathrm{~mm}$, the number of grains is approximately 6360. In each window, small grains, middle grains, and the large grains occupy approximately $57.7 \%$ of the volume and are subjected to the uniform probability distribution in the range of their sizes. Therefore, we set three kinds of sizes of the statistical windows: $\varepsilon^{3}=5 \mathrm{~mm}, \varepsilon^{2}=50 \mathrm{~mm}$, and $\varepsilon^{1}=100 \mathrm{~mm}$ to compute the equivalent mechanical parameter tensor with the different scale random grains.

Numerical results for the mechanical parameter tensor of a composite with only small, middle, and large rock grains are listed in Tables 6,7 , and 8 by the SMSA-FE algorithm. Tables 6-8 also show that the equivalent parameter tensor computed by the SMSA-FE algorithm possess the symmetrical, positive, and definite properties. The expected values of Young's modulus and Poisson's ratio for the different number of samples with three-scale random grains are shown in Table 9 and in Figure 2. Table 9 and Figure 2 show that the equivalent Young's modulus and Poisson's ratio are convergent.

A comparison of the numerical results for Young's modulus and Poisson's ratio by the SMSA-FE procedure, by the mixed volume method, and by the experiment method in the lab is also shown in Table 10. Table 10 also shows that Young's modulus and Poisson's ratios produced by SMSA-FE procedure are very close to that by the experiment method. It proves the SMSA-FE algorithm to be feasible and valid for predicting the effective modulus of the composites with random grains.

\section{Conclusion}

In this paper, we proved that the equivalent mechanical parameter tensor for the composite materials with multiscale random grains is convergent and obtained the error result by the finite element analysis. At the same time, we also prove that the equivalent parameter tensor matrix should satisfy the symmetric, positive, and definite property. 
Table 10: Equivalent mechanical parameters for a concrete by the different methods (GPa).

\begin{tabular}{lc}
\hline$E_{\text {SMSA-FE } 57.7 \%}=30.686221$ & $V_{\text {SMSA-FE } 57.7 \%}=0.193082$ \\
$E_{\text {test } 60 \%}=30.700000$ & $V_{\text {test } 60 \%}=0.198000$ \\
$E_{\text {average 60\% }}=50.600000$ & $V_{\text {average } 60 \%}=0.19000$ \\
\hline
\end{tabular}

Various test examples were solved by the SMSA-FE procedure. The numerical results show that the SMSA-FE procedure is feasible and valid and that these data satisfy the properties of the equivalent mechanical parameter tensor proved in the pervious sections.

The procedure can also be extended to other composite materials with random short fibers, random foams, random cavities, and so forth. Although we have given the specific steps, some theory results and numerical examples to carry out the SMSA-FE method and to illustrate the validity, the influence of shape, size, component, orientation, spatial distribution, and volume fraction of inclusions on inhomogeneous macromechanical properties, analyze the calculated results, and capture the information of microbehaviors to the macromechanical properties are also our important future work for the composite materials with random grains.

\section{Acknowledgments}

This work is supported by National Natural Science Foundation of China (NSFC) Grants (11072041), by State Key Laboratory of Structural Analysis for Industrial Equipment, Dalian University of Technology (GZ1005), by Hunan province Natural Science Foundation Grants of China (10JJ6065), by Scientific Research Starting Foundation for Returned Overseas Chinese Scholars, Ministry of Education, China (20091001), and by China Postdoctoral Science Foundation (20100480944).

\section{References}

[1] W. Voigt, Lehrbuch der Kristallphysik, Teubner, Leipzig, Germany, 1928.

[2] Z. Hashin and S. Shtrikman, "A variational approach to the theory of the elastic behaviour of multiphase materials," Journal of the Mechanics and Physics of Solids, vol. 11, no. 2, pp. 127-140, 1963.

[3] B. Budiansky, "On the elastic moduli of some heterogeneous materials," Journal of the Mechanics and Physics of Solids, vol. 13, no. 4, pp. 223-227, 1965.

[4] R. J. Farris, "Prediction of the viscosity of multimodal suspensions from unimodal viscosity data," Journal of Rheology, vol. 12, no. 2, pp. 281-301, 1968.

[5] J. Zuiker and G. Dvorak, "The effective properties of functionally graded composites-I. Extension of the mori-tanaka method to linearly varying fields," Composites Engineering, vol. 4, no. 1, pp. 19-35, 1994.

[6] R. Dierk, Computational Materials Science, Wiley-VCH, 1998.

[7] O. A. Oleinik, A. S. Shamaev, and G. A. Yosifian, Mathematical Problems in Elasticity and Homogenization, North-Holland, Amsterdam, The Netherlands, 1992.

[8] J. Z. Gui and H. Y. Yang, "A dual coupled method for boundary value problems of pde with coefficients of small period," Journal of Computational Mathematics, vol. 14, no. 2, pp. 159-174, 1996.

[9] Y. M. Poon, W. L. Luk, and F. G. Shin, "Statistical spherical cell model for the elastic properties of particulate-filled composite materials," Journal of Materials Science, vol. 37, no. 23, pp. 5095-5099, 2002.

[10] C. P. Tsui, C. Y. Tang, and T. C. Lee, "Finite element analysis of polymer composites filled by interphase coated particles," Journal of Materials Processing Technology, vol. 117, no. 1-2, pp. 105-110, 2001. 
[11] Y. P. Feng and J. Z. Cui, "Multi-scale FE computation for the structure of composite materials with small periodic configuration under condition of thermo-elasticity," Acta Mechanical Sinica, vol. 20, no. 1, pp. 54-63, 2004.

[12] W. M. He and J. Z. Cui, "A pointwise estimate on the 1-order approximation of $G_{x_{0}}^{\varepsilon}$," IMA Journal of Applied Mathematics, vol. 70, no. 2, pp. 241-269, 2005.

[13] L. Q. Cao, "Multiscale asymptotic method of optimal control on the boundary for heat equations of composite materials," Journal of Mathematical Analysis and Applications, vol. 343, no. 2, pp. 1103-1118, 2008.

[14] V. V. Jikov, S. M. Kozlov, and O. A. Oleinik, Homogenization of Differential Operators and Integral Functions, Springer, Berlin, Germany, 1994.

[15] T. Y. Hou and X. H. Wu, "A multiscale finite element method for elliptic problems in composite materials and porous media," Journal of Computational Physics, vol. 134, no. 1, pp. 169-189, 1997.

[16] E. Vanden-Eijnden, "Numerical techniques for multi-scale dynamical systems with stochastic effects," Communication Mathemtical Science, vol. 1, no. 2, pp. 385-391, 2003.

[17] W. Wang, D. Cao, and J. Duan, "Effective macroscopic dynamics of stochastic partial differential equations in perforated domains," SIAM Journal on Mathematical Analysis, vol. 38, no. 5, pp. 15081527, 2006.

[18] D. Duschlbauer, H. J. Böhm, and H. E. Pettermann, "Computational simulation of composites reinforced by planar random fibers: homogenization and localization by unit cell and mean field approaches," Journal of Composite Materials, vol. 40, no. 24, pp. 2217-2234, 2006.

[19] S. Kari, H. Berger, and U. Gabbert, "Numerical evaluation of effective material properties of randomly distributed short cylindrical fibre composites," Computational Materials Science, vol. 39, no. 1, pp. 198204, 2007.

[20] A. L. Kalamkarov, E. M. Hassan, A. V. Georgiades, and M. A. Savi, "Asymptotic homogenization model for 3D grid-reinforced composite structures with generally orthotropic reinforcements," Composite Structures, vol. 89, no. 2, pp. 186-196, 2009.

[21] A. Kalamkarov, I. Andrianov, and V. Danishevskyy, "Asymptotic homogenization of composite materials and structures," Applied Mechanics Reviews, vol. 62, no. 3, Article ID 030802, 20 pages, 2010.

[22] M. Wang and N. Pan, "Predictions of effective physical properties of complex multiphase materials," Materials Science and Engineering $R$, vol. 63, no. 1, pp. 1-30, 2008.

[23] M. Wang and N. Pan, "Elastic property of multiphase composites with random microstructures," Journal of Computational Physics, vol. 228, no. 16, pp. 5978-5988, 2009.

[24] P. W. Hao and D. L. Zhang, "Performance evaluation of polythene modified asphalt," in Asia-Pacific Reginal Meeting, Taipei, Taiwan, May 1996.

[25] Y. Li and J. Gui, "Two-scale analysis method for predicting heat transfer performance of composite materials with random grain distribution," Science in China, Series A, vol. 47, pp. 101-110, 2004.

[26] Y. Y. Li and J. Z. Cui, "The multi-scale computational method for the mechanics parameters of the materials with random distribution of multi-scale grains," Composites Science and Technology, vol. 65, no. 9, pp. 1447-1458, 2005.

[27] Y. Li, S. Long, and J. Cui, "Finite element computation for mechanics parameters of composite material with randomly distributed multi-scale grains," Engineering Analysis with Boundary Elements, vol. 32, no. 4, pp. 290-298, 2008.

[28] P. G. Ciarlet, The Finite Element Method for Elliptic Problems, Springer, Berlin, Germany, 1978. 


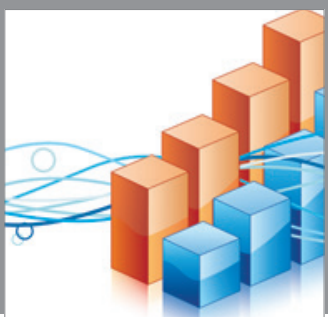

Advances in

Operations Research

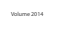

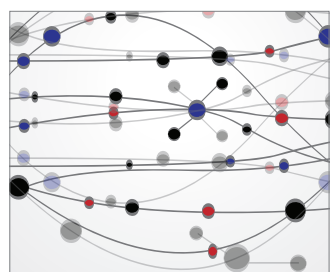

\section{The Scientific} World Journal
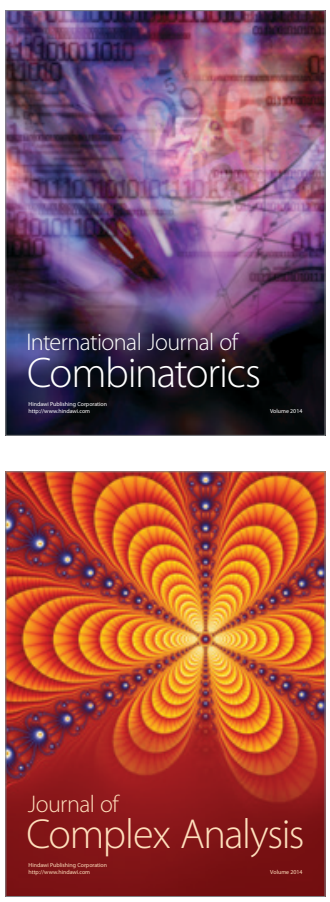

International Journal of

Mathematics and

Mathematical

Sciences
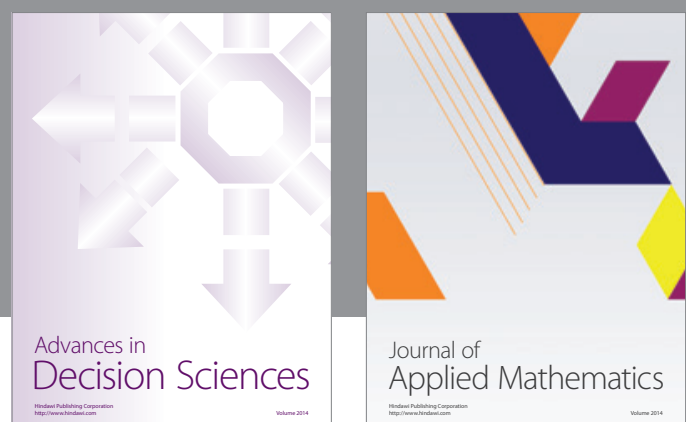

Journal of

Applied Mathematics
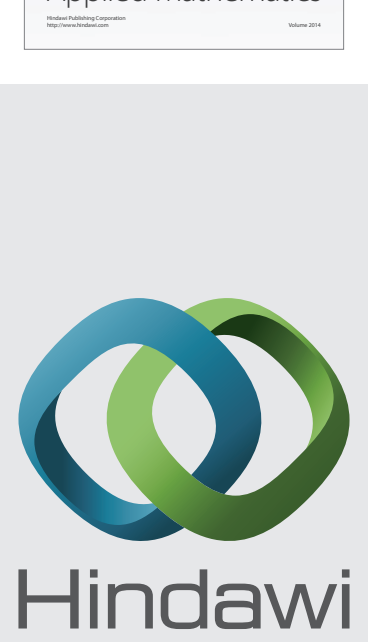

Submit your manuscripts at http://www.hindawi.com
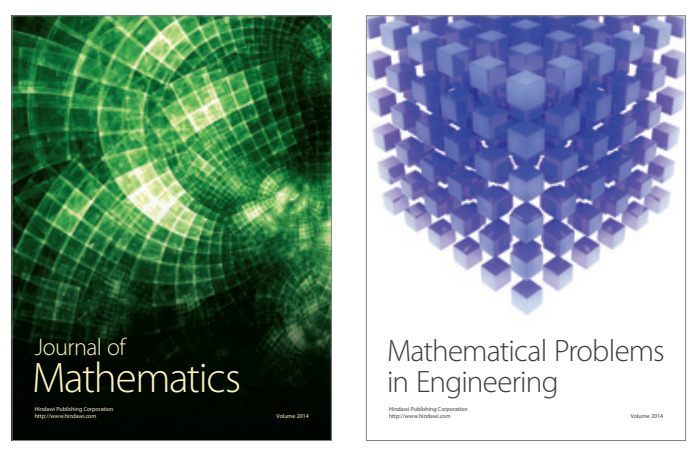

Mathematical Problems in Engineering
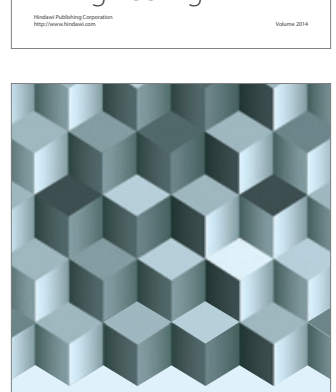

Journal of

Function Spaces
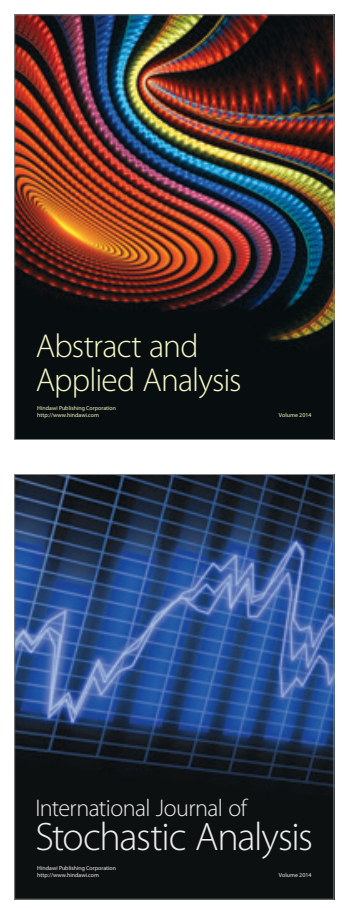

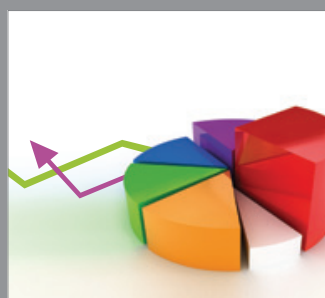

ournal of

Probability and Statistics

Promensencen
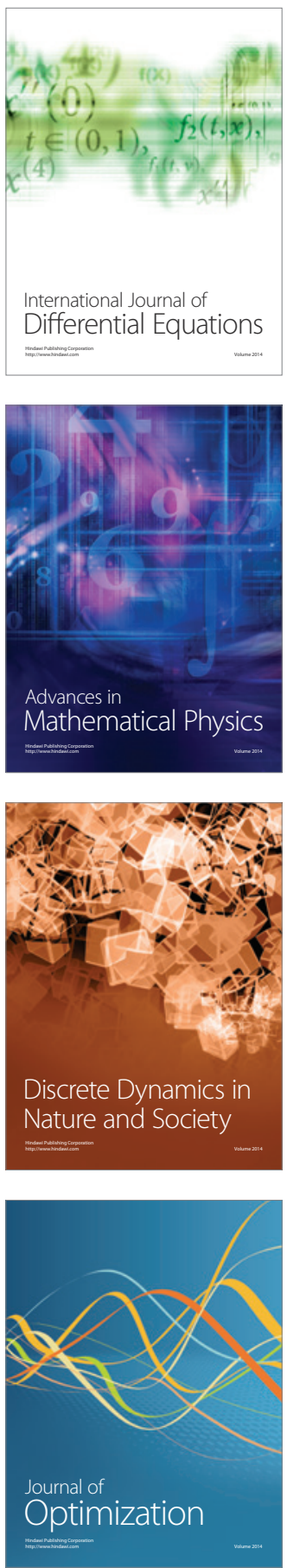